\title{
Rotational Motion Modelling for Numerical Analysis of Electric Machines
}

\author{
D. Marcsa $^{1,2}$ \\ ${ }^{1}$ Knorr-Bremse Commercial Vehicles, Research and Development Institute, \\ Engineering Calculation \\ Major u. 69., 1119, Budapest, Hungary \\ E-mail: daniel.marcsa@knorr-bremse.com \\ ${ }^{2}$ Széchenyi István University, Department of Automation \\ Egyetem tér 1., 9026, Győr, Hungary
}

\begin{abstract}
The paper presents a brief review of the movement modelling methods of electric machines and the two most common used torque calculation techniques. After the classification of single-layer moving band methods, a low computation cost and an easily realisable new variant of this movement modelling technique is proposed. To study the accuracy of proposed moving band technique equipped with Arkkio's method and Maxwell's stress tensor method for torque calculation an international benchmark problem used. Further, to check the applicability, the proposed method has been used to analyse a three-phase switched reluctance motor. The results of proposed method have been compared to analytical and numerical results.
\end{abstract}

Keywords: Rotational motion modelling, Finite element method, Torque calculation, Electric machine

\section{Introduction}

The numerical design and simulation of electromechanical actuators, and within that rotating electric machines are one of the main focus points of many researchers and research groups nowadays due to the hybrid and electric vehicles [1-4]. The aim of a numerical field calculation is to explore the overall behaviour of the analysing device, including all possible physical side effects simultaneously. To understand the behaviour of electric machines and to be able to intensify it, the accurate knowledge of the parameters governing physical effects must be known. The so-called coupled problem can distinguish between the single effects and may help to better understand the effects and their mode of operation [5-7]. 
A variety of numerical methods exists, but the most popular technique is finite element method (FEM) [8-10] among researchers in low-frequency electromagnetic applications. The mathematical description of the electromagnetic field can be derived from Maxwell's equations with appropriate boundary and interface conditions $[8,11]$. However, Maxwell's equations based formulations itself is only enough to time independent or steady-state simulations of rotating machines, it is not suitable for dynamic analysis.

The coupling plays an important role in the dynamic analysis of machine performance. The acceleration and mechanical transient of driving electric motor, so the propulsion performance is significant properties in the automotive industry. Consequently, it is important to take into consideration rotor movement in simulation via coupling [10-12]. There are many possibilities for stator-rotor connection and movement modelling, but most of them are computationally intensive or the implementation is difficult.

This article describes a brief stagger around the field of movement modelling in finite element method for electric machines. Moreover, I propose a simple and computationally efficient technique for modelling the rotational motion of the rotor. The torque calculation is also an important part of the dynamic simulation, therefore the paper briefly reviews the two most widely used methods. The applicability and performance of the proposed technique are analysed using an international benchmark problem and a three-phase switched reluctance motor. The combinations of presented torque calculation methods with movement modelling methods are also analysed via the simulation results.

\section{Rotational Motion Modelling Methods}

In this paper, the stator (fixed part) - rotor (mobile part) coupling classifies to the nature of the geometrical decomposition of the machine model. The field equation is applied to both parts, and the relative motion is taken into account in the air-gap, an ideal place to do it since it is non-ferromagnetic, non-conductive and without source. Based on it, one can distinguish between air-gap interface and sliding surface methods [10,12].

\subsection{Methods with Air-Gap Interface}

In methods of the air-gap interface, the air-gap or a part of it is considered as a separate domain with interfaces both to fixed and mobile part. The popularity of methods' group well shows the variants of air-gap discretisation technique as the macro-element method $[10,13]$, the moving band $[10,14,15,17,18]$, the boundary integral method [19] and the discontinuous Galerkin technique [20]. In the following, the macros-element and moving band method will be reviewed briefly.

First, the so-called macro- or air-gap element technique $[10,12,13]$ used to create the continuity between interfaces of stator and rotor meshed domains with an unmeshed air-gap region. This method is used analytical solution of $\nabla \times(\nabla \times \vec{A})=\overrightarrow{0}$ equation in 


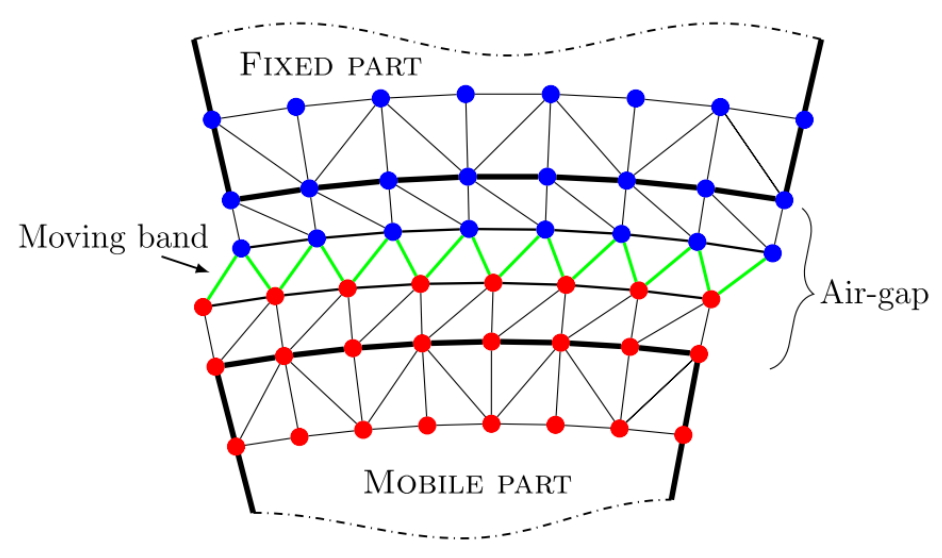

Figure 1. Single-layer moving band with triangular discretisation.

polar coordinate system which takes into account rotation of mobile part. The analytical solution is based on a truncated Fourier-series expansion of the $\vec{A}$ magnetic vector potential, where 100 to 200 harmonic terms give good accuracy [10]. Together with a high harmonic number, this technique introduces a dense part in the sparse finite element system of the equation which is also a drawback. An advantage of the air-gap element technique is that the results can be very accurate because of the high order of the field representation in the air-gap region.

However, to avoid the dense matrix block, and to use the long standing FEM solvers, proposed the moving band method (MB) [10,14-16] which is much more popular technique nowadays. In this method, the coupling between interfaces is commonly made by finite element placed in the air-gap, as it can be seen in Fig. 1. The simplest version of it, when each time step re-mesh the air-gap, however, this is not so computationally efficient. A more sophisticated way is to change the connectivity matrix between fixed and mobile part, while considered the element distortion.

\subsection{Methods with Sliding-Surface}

In sliding-surface techniques (SS), the rotational movement of the rotor is modelled at a common interface somewhere in the air-gap. The simplest variant of this group is the locked-step approach [21], where equidistant discretisation is applied, and the rotor rotates with an integer number of an angle between two adjacent nodes in each step. This is the conform version of sliding surface when the stator and rotor nodes are connected in each step, so the continuity maintains between the fixed and mobile parts. It is suitable for analysing the steady state of the machine but too severe for technical machine models. The dynamic modelling with the locked-step approach is computationally intensive because 


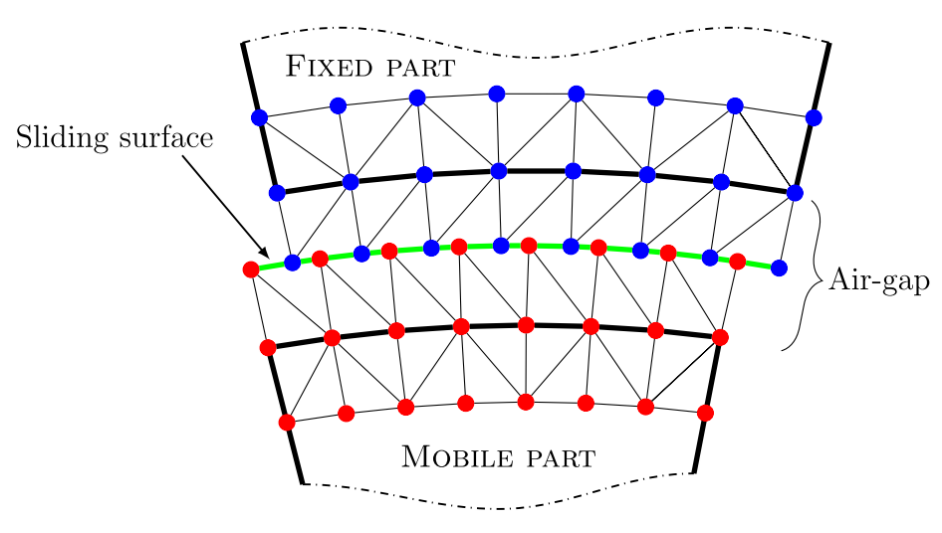

Figure 2. Sliding surface with nonconform mesh.

every time steps varies the length of time steps, namely the system matrix.

In general, the sliding surface techniques allow non-matching grids as you can see in Fig. 2. Therefore ensure the continuity of vector potential across the sliding surface an addition constraint. The most common techniques which are applied to enforce the continuity the polynomial (first or second order) interpolation [22], trigonometric interpolation $[18,23]$, Lagrange-multipliers [24,25] and mortar projection [26,27]. The common in all methods is one of the parts is the slave and the other one is the master surface, and the unknown variables of slave surface are described by master surface variables.

\subsection{Single-Layer Moving Band}

Based on the literature, the single-layer moving band (later moving band) is a widely used technique, but only in $[10,14,16,17]$ are mentioned or described the implementation of it. According to the descriptions, the following three types can be distinguished.

(1) Basic moving band [14]: This variant is the origin of the technique. This version is not dealing with the distortion of finite elements in the moving band. The operation of the technique, when the displacement is larger than the angle between two adjacent elements, than modified the connectivity matrix of moving band elements.

(2) Moving band with quadrilateral mesh $[10,16]$ : In this case, quadrilateral finite elements are used in the band, which are divided four triangular elements by the diagonals. The usage of triangles depends on the quality of elements and the angular displacement. To determine the quality of elements using a special formula which is based on the length of edges. 
(3) Moving band with trigonometric interpolation [17]: In this case, contrast to previous methods, the appropriate elements of system matrix will vary depending on the displacement. The number of finite elements decreases because of the use of trigonometric interpolation, however, the solution of the equation system is needed an iterative solver which incorporating Fast Fourier Transformation [18].

\subsubsection{Proposed Method}

The discretisation of proposed single-layer moving band boundaries in 2-D is equidistant with the same node and edge number. In this case also the connectivity matrix modified as variants (1) and (2). The base of this technique also the quadrilateral element as in (2), which is divided into two triangles depends on the $\alpha$ angle, as a measure of the distortion.

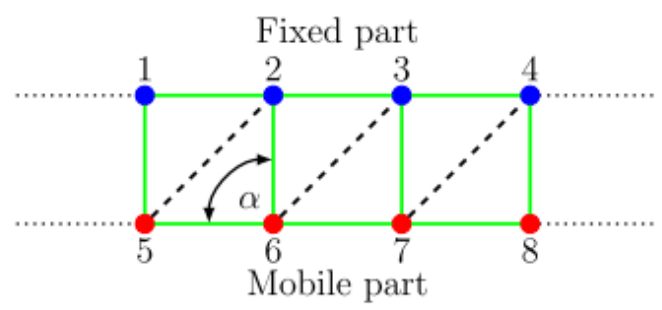

(a) Undeformed elements at base position.

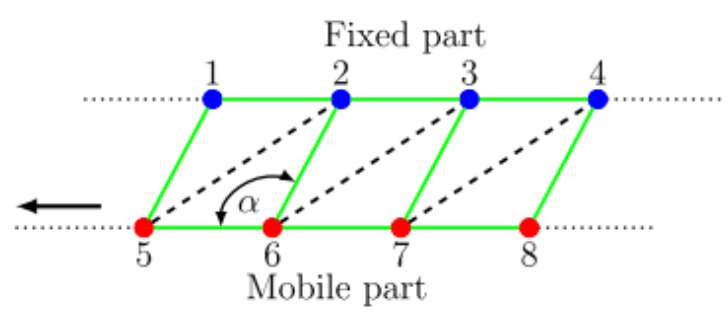

(b) The deformed elements in the band.

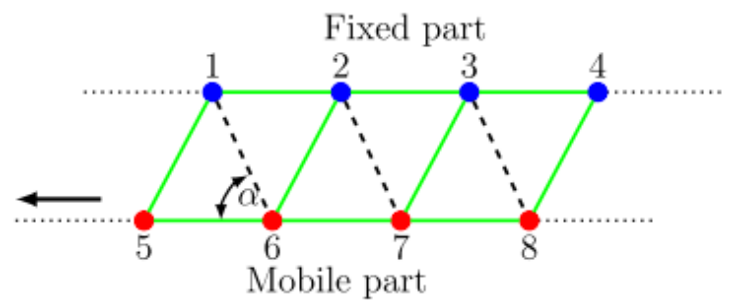

(c) The modification of band elements for better quality.

Figure 3. Proposed single-layer moving band method. 
This can be seen in Fig. 3. To determine all band elements quality is enough to examine only one element by the following equation

$$
\text { Quality factor }= \begin{cases}\operatorname{good}: & \text { if } \alpha \leq 90^{\circ} \\ \text { bad : } & \text { if } \alpha>90^{\circ}\end{cases}
$$

because all elements have the same shape.

The proposed method takes into account the element distortion, unlike the (1) version. The computation cost of elements quality is less in this case than in (2). Further, the implementation of it is much easier than the (3), and does not require special solver. The accuracy of the proposed method is not so high with the coarse mesh, however the usage of dense finite element mesh required for accurate torque calculation in the air-gap region, so the accuracy of this technique appropriate. This is supported by the used examples.

\section{Torque Calculation Methods}

The torque calculation also a cornerstone of rotational motion modelling as the before mentioned methods. In the followings, the two most common used torque calculation methods, Maxwell's stress tensor method (MST) [11,16, 25, 28] and method proposed by Arkkio (AM) [28,29] are briefly reviewed.

\subsection{Maxwell's stress tensor method}

The electromagnetic torque acting on the rotor of an electric machine may be calculated by integrating the Maxwell's stress tensor $\mathrm{d} \vec{F}$ along a line $\Gamma$ placing in the air-gap in two-dimensional case:

$$
\vec{T}_{e}=l \int_{\Gamma}(\vec{r} \times \mathrm{d} \vec{F}) \mathrm{d} \Gamma=l \int_{\Gamma}\left(\vec{r} \times\left[\mu_{0}(\vec{H} \cdot \vec{n}) \vec{H}-\frac{\mu_{0}}{2} H^{2} \vec{n}\right]\right) \mathrm{d} \Gamma,
$$

where $l$ is a depth of the domain in the $z$-direction, $\vec{r}$ is the position vector linking the rotating axis to the elements $\mathrm{d} \Gamma, \Gamma$ is a surface, which is placed around the air-gap, $\mu_{0}$ is the air magnetic permeability, $\vec{H}$ is the magnetic field strength, $H=|\vec{H}|$ is the absolute value of the magnetic field strength and $\vec{n}$ is the normal unit vector to the surface.

\subsection{The Method Proposed by Arkkio}

This method is a variant of the Maxwell's stress tensor method and consists in integrating the torque given by equation (2) in the whole surface (in 2-D) of the air-gap comprises between the radii $r_{R}$ and $r_{S}$. The torque is computed with the following equation:

$$
T_{e}=\frac{l}{\mu_{0}\left(r_{S}-r_{R}\right)} \int_{S} r B_{r} B_{\Theta} \mathrm{d} S
$$


where $S$ is the region between the radii $r_{R}$ and $r_{S} . B_{r}$ and $B_{\Theta}$ are the radial and tangential component of magnetic flux density, $r$ is the distance between the origin and the midpoint of the integration segment.

\section{Examples}

To check the validity of presented and proposed methods, an international benchmark problem of COMPUMAG Society, the T.E.A.M. 30a three-phase induction motor $[11,30]$ has been solved and compared the known analytic solution. This test problem used to analyse the combination of torque calculation methods with moving band and sliding surface technique. Further, to present the applicability of proposed method, here a threephase (6 stator and 4 rotor poles) switched reluctance motor [31] (SRM) has been solved and compared the simulation results.

\subsection{Problem T.E.A.M. 30a - Induction Motor}

As a test problem, a 2-D eddy current field analysis of three-phase test induction motor used. The detailed description of it including geometry and material parameters and the analytic solution can be found in [30]. A numerical solution of this problem a small above the synchronous speed can be seen in Fig. 4. This figure shows the magnetic vector potential distribution and the equipotential lines into the machine.

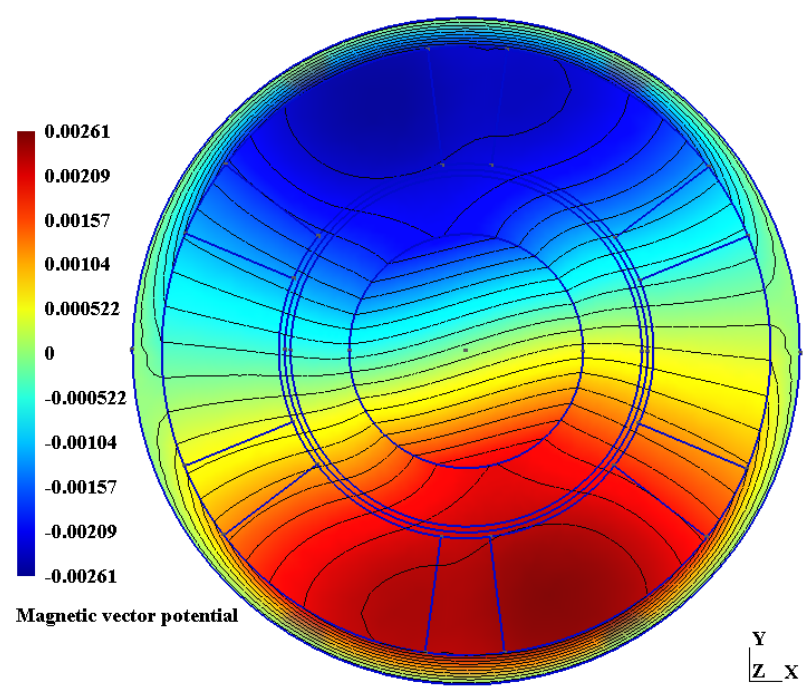

Figure 4. Field distribution in the T.E.A.M. 30a three-phase test motor at $400 \mathrm{rad} / \mathrm{s}$. 


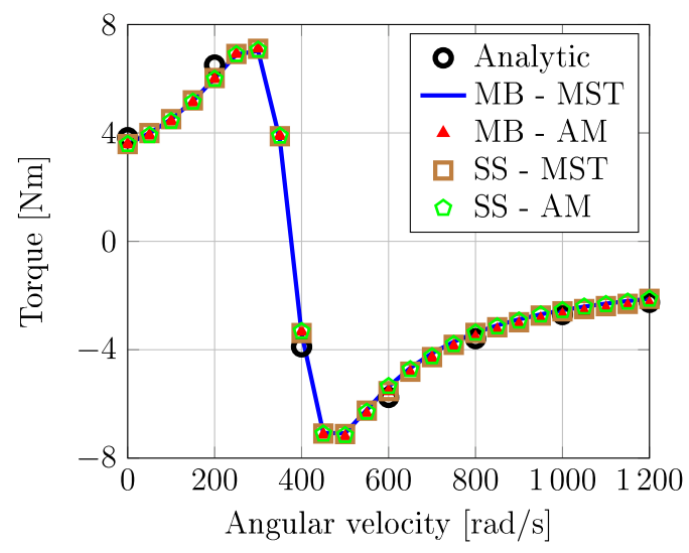

Figure 5. Calculated torque - angular velocity curves.

Fig. 5 shows the results of torque calculation. The analytic solution is given with $200 \mathrm{rad} / \mathrm{s}$ step within the range of analysis $(0, \ldots, 1200 \mathrm{rad} / \mathrm{s})$, wherein the numerical results are plotted with $50 \mathrm{rad} / \mathrm{s}$ step. The calculated numerical results show good agreement with the given analytic calculation. The biggest difference between analytic and numerical results is at $400 \mathrm{rad} / \mathrm{s}$. The reason for this big difference is the closure of synchronous speed $(\approx 377 \mathrm{rad} / \mathrm{s})$, where the torque-speed curve of the induction machine is steep. A small speed difference leads significant torque difference in this speed range.

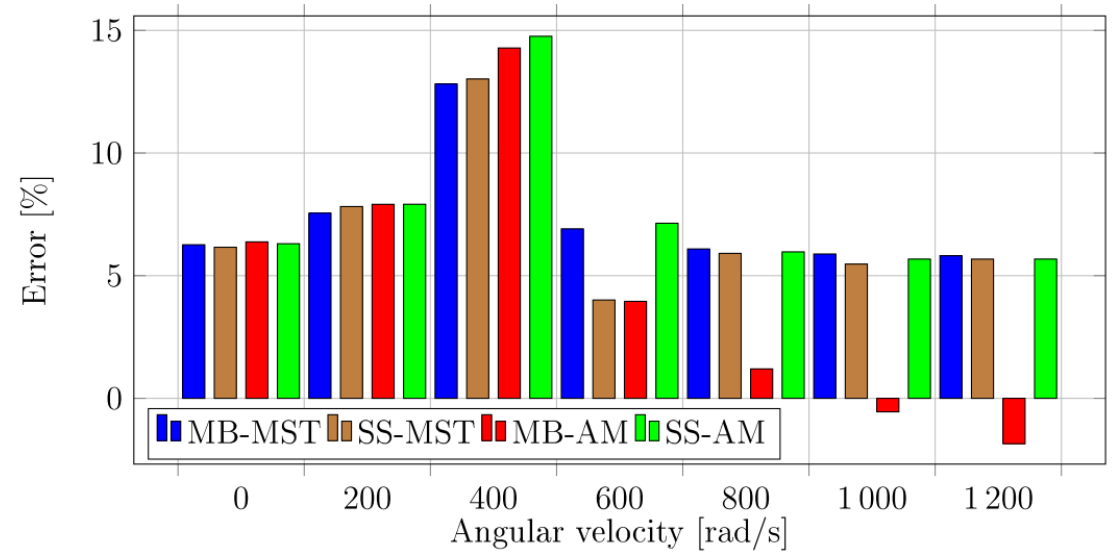

Figure 6. Bar plot of torque calculation error. 
The above statements also supported by the next figure, Fig. 6, where the torque calculation errors are summarised in a bar plot. The results of combined methods are approximately same, except the moving band with Arkkio's method above $600 \mathrm{rad} / \mathrm{s}$ angular velocity. Based on the results, the proposed moving band with Arkkio's method and sliding surface with Maxwell's stress tensor method have the best torque calculation performance, which agree with the literature [16, 25, 28].

\subsection{Switched Reluctance Motor}

After the benchmarking, a 6 stator pole and 4 rotor pole switched reluctance motor has been analysed by FEM. The geometry and material parameters of this application example are from examples of Agros 2D free finite element software [31]. Fig 7 shows the shape and results of the three-phase switched reluctance motor after the switching instance of winding excitation. The figure shows the absolute value of magnetic flux density distribution and equipotential lines.

Fig. 8 shows the calculated results, the torque in the function of mechanical angle. In this case, only the results of two combinations, the proposed moving band with AM and sliding surface with MST are analysed. The sliding surface results are used as the validation of implemented finite element code. The solution of Agros $2 \mathrm{D}$ is the reference solution, which is based on the co-energy variation torque calculation method $[10,28]$ with adaptive

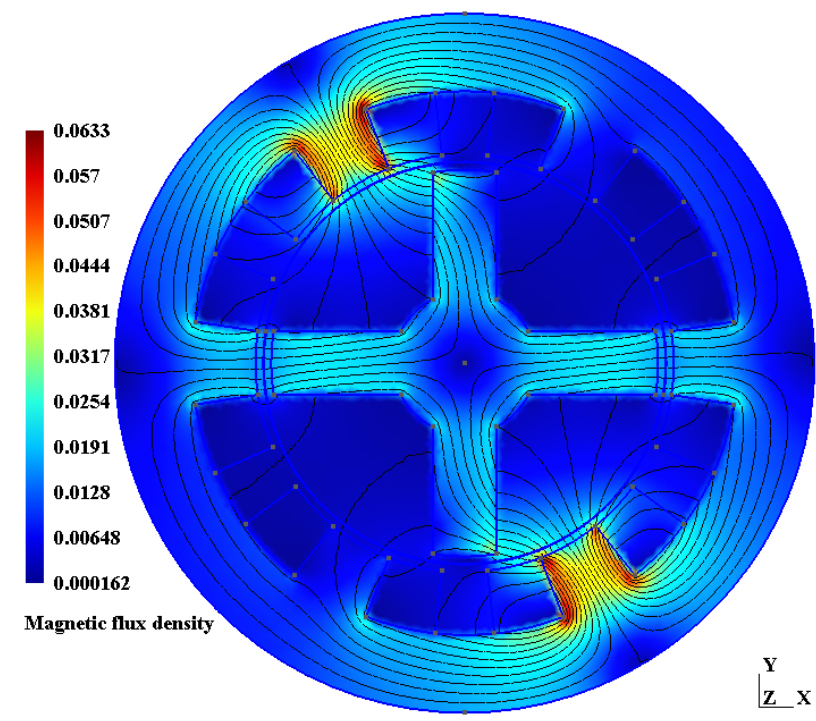

Figure 7. Field distribution in 6/4 switched reluctance motor. 


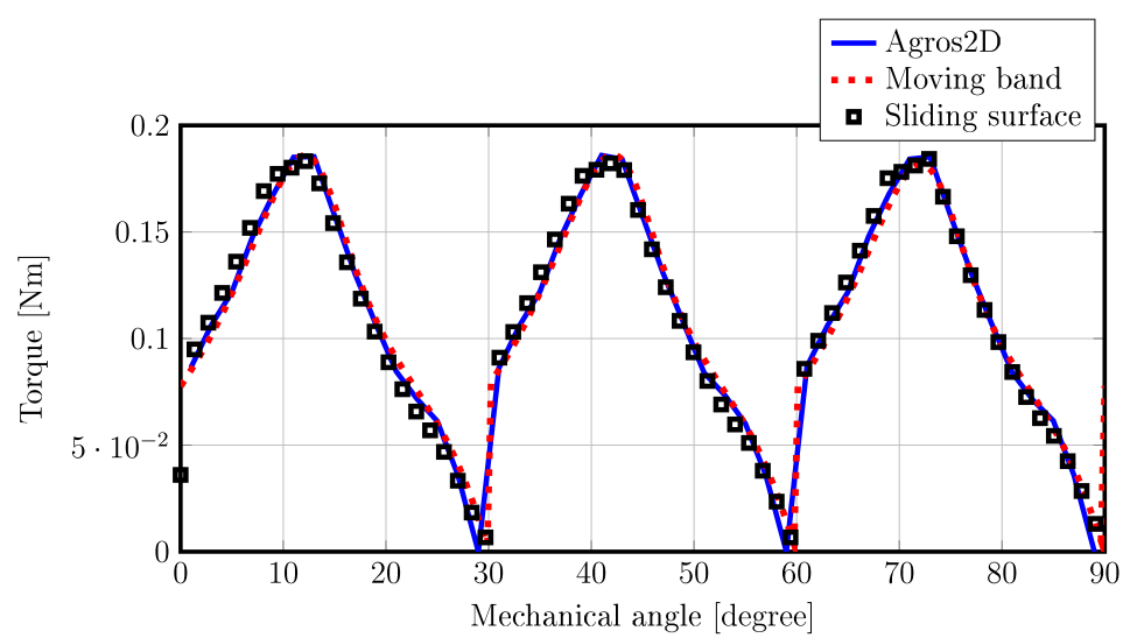

Figure 8. Torque curves in the function of mechanical angle of rotor.

mesh refinement technique. The results of presented methods show good agreement with the calculated torque from Agros2D, but the result with proposed method shows better relation.

\section{Conclusion and Future Works}

The paper has discussed rotational motion modelling and two most common used torque calculation techniques of electric machines. The available single-layer moving band methods have been classified based on the implementation and proposed a low computation cost and an easily realisable new variant of movement modelling technique. The accuracy and applicability of proposed single-layer moving band method are checked via two rotating machine examples.

It can be concluded that the accuracy of the proposed method is appropriate for electric machine modelling and almost the same than the sliding surface method. These conclusions also supported by simulated results, where the comparisons show good agreement.

The aim of the further research is to find a way to solve three-dimensional multiphysics problems including motional voltage-fed eddy-current field problems with the proposed movement modelling technique. 


\section{References}

[1] Sarigiannidis, A. G., Beniakar M. E., Kladas A. G.: Hybrid Analytical-FEM Methodology for Loss Evaluation in Traction Motors for Electric Vehicle Applications, in 2016 IEEE Conference on Electromagnetic Field Computation (CEFC), Miami, pp. $1-4,2016$

DOI: 10.1109/CEFC.2016.7816130

[2] Kim C. K.: A Novel Calculation Method on the Current Information of Vector Inverter for Interior Permanent Magnet Synchronous Motor for Electric Vehicle, IEEE Transactions on Magnetics, Vol. 50, No. 2, pp. 829-832, 2014

DOI: 10.1109/TMAG.2013.2279555

[3] Xiping, L., Ya, L., Zhangqi, L., Tao, L., Zhenhua, L.: Analysis and design of a high power density permanent magnet-assisted synchronous reluctance machine with low-cost ferrite magnets for EVs/HEVs, COMPEL - The international journal for computation and mathematics in electrical and electronic engineering, Vol. 35, No. 6, pp. 1949-1964, 2016

DOI: 10.1108/COMPEL-05-2016-0233

[4] Haiwei, C., Bo, G., Longya, X., Woongchul, C.: Optimal design of synchronous reluctance machine: A feasible solution to eliminating rare earth permanent magnets for vehicle traction applications, COMPEL - The international journal for computation and mathematics in electrical and electronic engineering, Vol. 33, No. 5, pp. $1569-1586,2014$

DOI: 10.1108/COMPEL-09-2013-0287

[5] Roger, D.: Electrical Machines ... a Challenge for the Year 00 ?, International Compumag Society Newsletter, Vol. 12, No. 1, pp. 5-7, 1999

[6] Kawase, Y.: Magnetic Field Analysis Coupled with Electric Circuit and Motion Equation, International Compumag Society Newsletter, Vol. 7, No. 3, pp. 12-16, 2000

[7] Preston, T. W.: Implementation of the Finite Element Method into an Industrial Design Environment, International Compumag Society Newsletter, Vol. 8, No. 3, pp. $2-8,2001$

[8] Kuczmann, M., Iványi, A.: The Finite Element Method in Magnetics, Akadémiai Kiadó, Budapest, 2008

[9] Luomi, J.: Finite Element Methods for Electrical Machines, Chalmers University of Technology, Gothenburg, 1993

[10] Bastos, J. P. A., Sadowski, N.: Electromagnetic Modeling by Finite Element Methods, Marcel Dekker, New York, 2003 
[11] Marcsa, D.: Finite Element Analysis of a Solid-Rotor Induction Machine, Acta Technica Jaurinensis, Vol. 3, No. 2, pp. 61-70, 2010

[12] De Gersem, H., Weiland, T.: Reformulation and Generalisation of the Air-Gap Element, International Compumag Society Newsletter, Vol. 12, No. 1, pp. 2—9, 2005

[13] Razek, A. A., Coulomb, J. L., Féliachi, M., Sabonnadiére J. C.: A Concept of an Air-Gap Element for the Dynamic Analysis of the Electromagnetic Field in Electric Machines, IEEE Transactions on Magnetics, Vol. 18, No. 2, pp. 655—659, 1982 DOI: 10.1109/TMAG.1982.1061898

[14] Davat, B., Ren, Z., Lajoie-Mazenc, M.: The Movement in Field Modeling, IEEE Transactions on Magnetics, Vol. 21, No. 6, pp. 2296-2298, 1985

DOI: 10.1109/TMAG.1985.1064185

[15] Dular, P., Geuzaine, C., Ferreira da Luz, M. V., Sadowski, N., Bastos, J. P. A.: Connection Boundary Conditions with Different Types of Finite Elements Applied to Periodic Conditions and to the Moving Band, COMPEL - The international journal for computation and mathematics in electrical and electronic engineering, Vol. 20, No. 1, pp. 109-119, 2001

DOI: $10.1108 / 03321640110359796$

[16] Sadowski, N., Lefévre, Y., Lajoie-Mazenc, M., Cros, J.: Finite Element Torque Calculation in Electrical Machines While Considering the Movement, IEEE Transactions on Magnetics, Vol. 28, No. 2, pp. 1410-1413, 1992

DOI: $10.1109 / 20.123957$

[17] Demenko, A.: Movement Simulation in Finite Element Analysis of Electric Machine Dynamics, IEEE Transactions on Magnetics, Vol. 32, No. 3, pp. 1553-1556, 1996 DOI: $10.1109 / 20.497547$

[18] De Gersem, H., Ion, M., Wilke, M., Weiland, T., Demenko, A.: Trigonometric Interpolation at Sliding Surface and in Moving Bands of Electrical Machine Models, COMPEL - The international journal for computation and mathematics in electrical and electronic engineering, Vol. 25, No. 1, pp. 31-42, 2006

DOI: $10.1108 / 03321640610634308$

[19] Salon, S. J., Schneider, J. M.: A Hybrid Finite Element - Boundary Integral Formulation of the Eddy Current Problem, IEEE Transactions on Magnetics, Vol. 18, No. 2, pp. 461-466, 1982

DOI: 10.1109/TMAG.1982.1061891

[20] Alotto, P., Bertoni, A., Perugia, I., Schötzau, D.: Discontinuous Finite Element Methods for the Simulation of Rotating Electrical Machines, COMPEL - The international journal for computation and mathematics in electrical and electronic engineering, Vol. 20, No. 2, pp. 448-462, 2001

DOI: $10.1108 / 03321640110383320$ 
[21] Preston, T. W., Reece, A. B. J., Sangha, P. S.: Induction Motor Analysis by TimeStepping Technique, IEEE Transactions on Magnetics, Vol. 24, No. 1, pp. 471-474, 1988

DOI: $10.1109 / 20.43959$

[22] Perrin-Bit, R., Coulomb, J. L.: A Three Dimensional Finite Element Mesh Connection for Problems Involving Movement, IEEE Transactions on Magnetics, Vol. 31, No. 3, pp. $1920-1923,1995$

DOI: $10.1109 / 20.376415$

[23] De Gersem, H., Weiland, T.: Harmonic Weighting Functions at the Sliding Interface of a Finite-Element Machine Model Incorporating Angular Displacement, IEEE Transactions on Magnetics, Vol. 40, No. 2, pp. 545-548, 2004

DOI: $10.1109 /$ TMAG.2004.824616

[24] Rodger, D., Lai H. C., Leonard, P. J.: Coupled Elements for Problems Involving Movement, IEEE Transactions on Magnetics, Vol. 26, No. 2, pp. 548-550, 1990 DOI: $10.1109 / 20.106375$

[25] Antunes, O. J., Bastos, J. P. A., Sadowski, N.: Comparison Between Torque Calculation Methods in a Non-Conforming Movement Interface, COMPEL-The International Journal for Computation and Mathematics in Electrical and Electronic Engineering, Vol. 27, No. 1, pp. 27-36, 2008

DOI: $10.1108 / 03321640810836609$

[26] Rodger, D., Lai, H. C., Leonard, P. J.: Coupled Elements for Problems Involving Movement, IEEE Transactions on Magnetics, Vol. 26, No. 2, pp. 548-550, 1990 DOI: $10.1109 / 20.106375$

[27] Buffa, A., Maday, Y., Rapetti, F.: Calculation of Eddy Currents in Moving Structures by a Sliding Mesh-Finite Element Method, IEEE Transactions on Magnetics, Vol. 36, No. 4, pp. $1356-1359,2000$

DOI: $10.1109 / 20.877690$

[28] Henrotte, F.: Handbook for the Computation of Electromagnetic Forces in a Continuous Medium, International Compumag Society Newsletter, Vol. 11, No. 2, pp. 2-8, 2004

[29] Arkkio, A.: Analysis of Induction Motor Based on the Numerical Solution of the Magnetic Field and Circuit Equations, PhD thesis, Helsinki University of Technology, 1987

[30] Davey, K. R.: Induction Motor Analysis-International TEAM Workshop Problem 30 [Online]. Available: http://www.compumag.co.uk

[31] Agros2D - free finite element software [Online]. Available: https://www.agros2d.org/ 